\title{
25 Research Square \\ Incidence and Risk Factors for Bilateral \\ Nephrolithiasis: A Large Case-retrospective Study
}

\section{Xiong Yang}

Tianjin Institute of Urology, The Second Hospital of Tianjin Medical University

Zhi Li

Tianjin Institute of Urology, The Second Hospital of Tianjin Medical University

\section{Shiyong Qi}

Tianjin Institute of Urology, The Second Hospital of Tianjin Medical University

\section{Linguo Xie}

Tianjin Institute of Urology, The Second Hospital of Tianjin Medical University

\section{Qiduo Shi}

Tianjin Institute of Urology, The Second Hospital of Tianjin Medical University

Chunyu Liu ( $\nabla$ dr.chunyuliu@hotmail.com )

Tianjin Institute of Urology, The Second Hospital of Tianjin Medical University

\section{Research Article}

Keywords: Nephrolithiasis, Metabolic syndrome, Gout, Diabetes mellitus, Hypertension

Posted Date: April 22nd, 2021

DOl: https://doi.org/10.21203/rs.3.rs-414196/v1

License: (c) (1) This work is licensed under a Creative Commons Attribution 4.0 International License.

Read Full License 


\section{Abstract}

To determine the incidence and risk factors of bilateral kidney stones. Utilized the retrospective analysis method on demographic characteristics and clinical data of patients with renal stones in the Second Hospital of Tianjin Medical University. Grouped patients into unilateral and bilateral renal stones according to preoperative imaging and ultrasound examination. Univariate and multivariate analysis methods were used to evaluate the factors that may cause bilateral stones. The study included 7587 patients with kidney stones in total, of whom 4983 had unilateral kidney stones (including 2719 left stones and 2264 right stones), and 2604 had bilateral kidney stones (34.3\%). By comparing the unilateral stones group with the bilateral stones group, the univariate analysis demonstrated that weight, body mass index (BMI), history of nephrolithiasis, diabetes mellitus (DM), hypertension, gout, and the maximal stone diameter had statistical significance. Binary logistic regression multivariate analysis demonstrated that BMI, history of nephrolithiasis, diabetes mellitus, hypertension, gout, and the maximal stone diameter were independent risk factors for bilateral urinary stones. This study shows that $34.3 \%$ of patients with kidney stones were diagnosed as having bilateral kidney stones; BMI and the maximal stone diameter are positively correlated with the incidence of bilateral kidney stones; Patients with a history of nephrolithiasis, diabetes, hypertension, and gout have a significantly higher risk of having bilateral kidney stones.

\section{Introduction}

Urolithiasis is one of the common diseases of the urinary system. As people's living environment and eating habits are constantly changing, the prevalence rate of urolithiasis worldwide increases year by year $^{1,2}$. According to the latest epidemiological studies of kidney stones in mainland China, the incidence of kidney stones in mainland China was 5.95\% from 1991 to $2000,8.86 \%$ from 2001 to 2010, and 10.63\% from 2011 to present, showing a significant upward trend ${ }^{3}$. Besides, kidney stones are a disease with a high recurrence rate. About $50 \%$ of patients relapse within 5-10 years after treatment, and the recurrence rate of stones within 20 years is close to $75 \%^{4}$. Previous studies have shown that various factors are involved in forming kidney stones, including urinary tract obstruction, infection, foreign bodies, drug use, and metabolic disorders. However, due to the improvement of people's awareness of medical treatment and early drug intervention, the proportion of infectious factors in urinary tract stones formation decreases ${ }^{5}$. Nevertheless, various clinical studies related to kidney stones' formation have received increasing attention from clinicians in recent years.

In recent years, an increasing number of studies have illustrated that chronic diseases such as metabolic syndrome (MetS), hypertension, diabetes mellitus (DM), obesity, gout, and chronic kidney disease are significantly related to kidney stones' occurrence and development ${ }^{6-10}$. Shimizu, $T$ et al. reported that hyperuricemia is the leading cause of gout. About $34 \%$ of gout patients have kidney stones, and more than $50 \%$ of them have bilateral kidney stones ${ }^{11}$. As a metabolic disease, it has been initially confirmed that gout can promote the onset of kidney stones, especially bilateral kidney stones. There are few reports 
on the correlation between other metabolic diseases and the onset of bilateral kidney stones. This study retrospectively analyzed the clinical data of patients with kidney stones who received treatment from the Department of Urology, Tianjin Medical University Second Hospital from January 2017 to June 2020, to explore the factors affecting the incidence of bilateral kidney stones, guide the choice of clinical treatment plan aiming at reducing the formation and recurrence of stones.

\section{Results}

\section{Demographic characteristics and clinical data}

In this study, among 7587 patients with kidney stones, there were 2604 cases of bilateral kidney stones, and the proportion of bilateral kidney stones was $34.3 \%$; The average age is $48.2 \pm 13.1$ years; the average $\mathrm{BMI}$ is $25.96 \pm 3.53 \mathrm{~kg} / \mathrm{m}^{2}$; gender distribution: male VS female $6043 \mathrm{VS} 1544$; data are shown in Table 1 .

\section{Univariate analysis of unilateral and bilateral kidney stones}

There are significant differences between the bilateral kidney stone group and the unilateral kidney stone group regarding body weight, $\mathrm{BMl}$, and maximum stone diameter. Regarding the past medical history, there are significant differences between the history of kidney stones, hypertension, diabetes, and gout between the groups of bilateral and unilateral kidney stones. The results are statistically significant $(P<0.001)$, as showed in Table 2.

\section{Univariate analysis of left, right, and bilateral kidney stones}

There were statistically significant clinical data in the unilateral and bilateral kidney stone groups, and the comparison between the left, right, and bilateral kidney stone groups was also statistically significant $(P<0.001)$. There was no significant difference in whether the stone was on the left or the right in the unilateral kidney stone group, compared with bilateral kidney stones. Besides, the study also found no significant correlation between the affected side of the stone and whether the stone is more frequent in the left and right kidney stone groups. Also, the difference is not statistically significant, as shown in Table 3.

\section{The Binary logistic regression univariate and multivariate analysis}

We first compared the unilateral and bilateral renal stone groups through binary logistic regression univariate analysis and then used logistic regression multivariate analysis to analyze further the statistically significant indicators obtained. The results indicated that BMI, the history of nephrolithiasis, history of diabetes, history of hypertension, history of gout, and the maximal stone diameter were independent risk factors for forming bilateral kidney stones, as shown in Table 4.

\section{Discussion}


With the change in living habits, and the improvement of imaging technology, the incidence and prevalence of urolithiasis are increasing. Particularly, bilateral urinary stones pose a considerable challenge to clinicians ${ }^{12}$. Bilateral urinary tract stones affect the urinary tract's patency, thereby affecting the change of the internal urine environment and the damage of kidney function ${ }^{11}$. Therefore, for patients with bilateral kidney stones, early detection will not only help clinicians formulate the best treatment plan, but also help patients prevent the recurrence of kidney stones.

In this study, $34.3 \%$ of all patients with kidney stones were diagnosed with bilateral kidney stones, which was higher than previous reports ${ }^{13}$. Such an increase may be attributed to the changes in people's living habits and the increasing incidence of metabolic diseases in recent years. Our research indicates that compared with patients with normal metabolism, patients with metabolic-related diseases have a higher incidence of bilateral kidney stones. The multivariate analysis results and univariate analysis indicated that BMI, history of kidney stones, history of diabetes, history of hypertension, history of gout, and the maximal stone diameter are independent risk factors for forming bilateral kidney stones. Among the patients with bilateral kidney stones, there were 1937 (25.5\%) obese patients $\left(\mathrm{BMI} \geq 24 \mathrm{~kg} / \mathrm{m}^{2}\right), 795$ (10.5\%) patients with hypertension, and 470 (6.2\%) patients with diabetes. According to previous research reports, the incidence of metabolic syndrome and urinary tract stones is significantly positively correlated $^{8,14}$. Metabolic syndrome includes hypertension, insulin resistance, obesity, and dyslipidemia. The definition of metabolic syndrome varies according to different standards. The internationally recognized threshold value of the American Diabetes Association IDF standard (by race and gender) is that the three or more following terms are met: (1) Fasting triglyceride content is $150 \mathrm{mg} / \mathrm{dL}$ and above; (2) Systolic blood pressure is $130 \mathrm{mmHg}$ and above, and or diastolic blood pressure is $85 \mathrm{mmHg}$ and above; (3) Fasting blood glucose content is $100 \mathrm{mg} / \mathrm{dL}$ and above; (4) High-density lipoprotein (HDL) is $40 \mathrm{mg} / \mathrm{dL}$ and below for men and $50 \mathrm{mg} / \mathrm{dL}$ and below for women; (5) Waist circumference: $90 \mathrm{~cm}$ and above for men and $80 \mathrm{~cm}$ and above for women ${ }^{15}$. Although many studies have confirmed that metabolic syndrome can promote the onset of urinary calculi in recent years, this study initially confirmed that metabolic syndrome is significantly positively correlated with the onset of bilateral kidney stones. Besides, this study also explored the correlation between the affected side of kidney stones and the number of stones, and the study results showed that the two were not statistically significant.

$\mathrm{BMI}$ can well represent the relationship between weight and height, and BMI can also reflect the state of fat storage. Our research demonstrates that the average BMI of patients with bilateral kidney stones $\left(26.23 \pm 3.53 \mathrm{~kg} / \mathrm{m}^{2}\right)$ is significantly higher than that of patients with unilateral kidney stones $(25.82 \pm$ $3.52 \mathrm{~kg} / \mathrm{m}^{2}$ ). This is consistent with reports written by Milicevic, $\mathrm{S}$ et al. and Scales CD Jr et al. ${ }^{1,7}$. That is, obesity, overweight, and kidney stones are related, and as BMI increases, urinary calcium and uric acid excretion increases, and the incidence and recurrence rate of urolithiasis are also rising. Antonelli, J A reported that compared with the average human, the incidence of urolithiasis in obese and overweight patients was as high as $75 \%{ }^{16}$. This may be the same reason why patients with higher BMI often formulate bilateral kidney stones. Therefore, patients can reduce the incidence of urolithiasis by adjusting diet, strengthening exercise, and lowering BMI. 
Kabeya, Y, and Weinberg, A E reported that kidney stones' incidence and recurrence rate were significantly higher in patients with than without $\mathrm{DM}^{17,18}$. The study by Devasia, $\mathrm{D}$ et al. reported that in patients with diabetes and urinary calculi, serum adiponectin levels and the content of calcium and phosphate in urine were significantly higher than those in patients with diabetes without urinary calculi ${ }^{19}$. Our research results show that compared with non-diabetic patients, diabetic patients are more likely to develop bilateral kidney stones. The reason may be related to two underlying mechanisms: one is that in insulinresistant diabetic patients, due to the change of uric acid clearance rate, acid urine is often produced, and long-term changes in the internal environment of urine can promote the formation of uric acid stones ${ }^{20}$; The other is that diabetic patients are significantly higher than non-diabetic patients in terms of gastrointestinal absorption of oxalate and endogenous metabolism to produce oxalate ${ }^{21}$.

Hypertension can increase the reabsorption of water, sodium, and calcium by the kidneys, leading to multiple atherosclerosis in the kidneys, which significantly increases the risk of calcium stones and uric acid stones. Our study results show that patients with a history of hypertension have a significantly higher incidence of bilateral kidney stones. This is consistent with the reports of Kohjimoto $Y$ et al. and Cappuccio FP et al.: Compared with normal people, patients with hypertension have a significantly increased risk of kidney stones ${ }^{22,23}$. Therefore, patients can effectively control high blood pressure and prevent calcium stone formation through a low-sodium diet and eating more fruits and vegetables. Recent studies have reported that thiazide diuretics can effectively relieve hypertension and prevent stones' formation for patients with ineffective dietary adjustments ${ }^{24}$.

In summary, we speculate that metabolic-related diseases, especially metabolic syndrome, may be an independent risk factor for bilateral kidney stones, and the reason is related to changes in urine composition. Research by Cho, ST et al. reported: Compared with patients without MetS, urine PH, citrate, uric acid, and calcium in patients with MetS were significantly lower, while the content of uric acid in the blood was significantly increased. Patients with one characteristic of MetS have a risk of stones of $10.2 \%$, and those with four characteristics of MetS have a risk of stones of $30.4 \%$. Although our study did not further clarify whether the superposition of various metabolic factors will further promote the onset of bilateral urinary tract stones, it preliminarily confirmed the correlation between MetS and bilateral urinary tract stones.

Gout is one of the important complications of hyperuricemia. Our results show that the proportion of bilateral kidney stones is $58.2 \%$ in all patients with gout and kidney stones. This is consistent with reports by Shimizu, T: About $34 \%$ of gout patients have urinary tract stones, and more than $50 \%$ of them have bilateral urinary tract stones ${ }^{11}$. Due to the low $\mathrm{pH}$ value of urine in patients with gout, the solubility of uric acid crystals in urine is low, and uric acid precipitates in the urinary tract to form stones ${ }^{26}$. Another generally accepted explanation is that in the urine of patients with gout, uric acid crystals are used as the core to form heterogeneous nucleation with uric acid crystals as the core, and the urate and oxalate crystals grow epitaxially, thereby forming mixed urinary tract stones ${ }^{27}$. We believe that these mechanisms may also promote the formation of bilateral urinary tract stones. 
Regarding the correlation between the past history of kidney stones and bilateral kidney stones, our research results show that the first kidney stones found are more common in unilateral kidney stones, while patients with recurrent kidney stones are more common in bilateral kidney stones. The reasons may be related to lifestyle habits such as low water intake, a high purine diet, and taking particular drugs ${ }^{26,28}$. If these habits contributing to kidney stones' pathogenesis are not effectively corrected, it may lead to the formation of bilateral kidney stones.

Additionally, our research also found that the stones' diameter is positively correlated with the incidence of bilateral kidney stones. Previous studies by Wen JG et al. reported that the maximum diameter of stones is related to renal failure, resulting in decreased renal excretion of uric acid and calcium ${ }^{29}$. To a certain extent, this can explain our research results. Simultaneously, the maximal diameter of a stone is positively correlated with the stone's duration, and these factors may promote the formation of bilateral kidney stones. We also compared the relationship between the stone affected side (left/right) and the number of stones (single/multiple), but the study results showed no significant correlation between the two. At present, there are few reports on related studies between the two, and a large randomized controlled study is still needed for further demonstration.

The shortcomings of this study: First of all, as to whether the stone composition is related to the formation of bilateral kidney stones, relevant data collection and statistical analysis were not carried out; Secondly, because the content of uric acid and blood lipids is a dynamic process, and the content of both is also related to the patient's recent diet and drug control, it is impossible to obtain an optimal predictive value for the formation of bilateral kidney stones; Third, due to the diversity of Chinese people's dietary habits and the diversity of medications for chronic diseases, the data on dietary habits and medications have not been collected and included for analysis.

\section{Conclusion}

This study shows that in patients with kidney stones, the rate of bilateral kidney stones is $34.3 \%$. BMI, history of kidney stones, history of diabetes, history of hypertension, history of gout, and the largest diameter of stones are independent risk factors for the formation of bilateral kidney stones. The patient's kidney stones on the affected side (left/right) and the number of stones (single/multiple) were not statistically significant for bilateral kidney stones. The incidence of bilateral kidney stones is mostly related to metabolic-related diseases. It is recommended to improve the comprehensive metabolic assessment of patients with kidney stones, adjust their living habits, and prevent the recurrence of kidney stones.

\section{Materials And Methods}

\section{Demographic characteristics}


The demographic characteristics and clinical data of 7587 patients with kidney stones in the Department of Urology, Second Hospital of Tianjin Medical University from January 2017 to June 2020, were selected. The stones' locations are as follows; 4983 cases of unilateral kidney stones were included, of which 2719 cases were on the left side, 2264 cases were on the right side, and 2604 cases were bilateral kidney stones. Among unilateral kidney stones, there were 1649 cases of single stones on the left, 1070 cases of multiple stones on the left, 1424 cases of single stones on the right, and 840 cases of multiple stones on the right.

\section{Inclusion criteria}

(1) Attended to the urology department of our hospital, ultrasound examination and/or abdominal computed tomography (CT) examination confirmed that the patient had kidney stones; (2) Patients with complete clinical data.

\section{Exclusion criteria}

(1) Renal pelvic ureteral stricture or congenital malformations of the ureter; (2) History of open kidney and ureter surgery or laparoscopic surgery; (3) History of abdominal radiotherapy; (4) History of retroperitoneal fibrosis; (5) Patients who have a history of repeated urinary tract infections and have not been well controlled; (6) Patients with incomplete clinical data.

\section{Identify stones}

Two experienced sonographers performed an ultrasound examination on the same patient with the same type of instrument and $3.0-5.0 \mathrm{MHz}$ ultrasound probe and recorded the size, location, and the number of stones. The longest axis of the stone is defined as the maximum stone diameter. Due to the limitations of ultrasound equipment, stones were defined as particles $\geq 5 \mathrm{~mm}$. If the two doctors' diagnosis results are quite different, a non-enhanced spiral CT scan with a thickness of $2 \mathrm{~mm}$ is performed ${ }^{30}$.

\section{Statistical analysis}

Statistical analysis was conducted with SPSS 23.0 software. Age, gender, height, weight, body mass index (BMI), history of nephrolithiasis, diabetes mellitus (DM), hypertension, cerebrovascular disease (CVD), stroke, liver and gallbladder disease, gastrointestinal disease, respiratory disease, chronic kidney disease, orthopedic disease, tumor disease, gout, other diseases, and the maximal stone diameter were statistically described. T-test, Chi-square test, and Mann-Whitney U rank-sum test were applied in unilateral and bilateral renal stone groups; One-way ANOVA test, chi-square test, and Kruskal-Wallis $\mathrm{H}$ test were applied in left, right, and bilateral kidney stone groups. Odds ratios (ORs) and 95\% confidence intervals (Cls) of the unilateral and bilateral renal stone groups were obtained using binary logistic regression in univariable and multivariable analyses.

\section{Declarations}


Acknowledgments: This work was supported by grants from National Natural Science Foundation of China (grant number: 81700616 and 82070725), the Science and Technology Project of Tianjin (grant number: 18PTLCSY00020 and 17ZXMFSY00060), and the Education Commission Research Project of Tianjin (grant number: 2017KJ208 and 2017KJ206).

Author contributions X.Y. performed protocol and project development, data collection and management, data analysis, manuscript writing. Z.L. performed data management and analysis, manuscript writing. S.Y.Q. performed data collection and management. L.G.X. performed data collection and management. Q.D.S. performed protocol development, data management, manuscript editing. C.Y.L. performed protocol and project development, data collection and management, manuscript editing. All authors reviewed the manuscript.

Conflict of interest: All the authors declare that they have no conflict of interest.

\section{Data Availability}

The data used to support the findings of this study are available upon request to the corresponding author.

Ethical standards $\square$ All studies have been approved by the Ethics Committee of the Second Hospital of Tianjin Medical University and have, therefore, been performed in accordance with the ethical standards laid down in the 1964 Declaration of Helsinki and its later amendments. All written consents from patients were waived due to the retrospective nature of the current study.

\section{References}

1. Scales, C. J., Smith, A. C., Hanley, J. M. \& Saigal, C. S. Prevalence of kidney stones in the United States. EUR UROL. 62, 160-165 (2012).

2. Heers, H. \& Turney, B. W. Trends in urological stone disease: A 5-year update of hospital episode statistics. BJU INT. 118, 785-789 (2016).

3. Wang, W. et al. Prevalence of kidney stones in mainland China: A systematic review. Sci Rep. 7, 41630 (2017).

4. Moe, O. W. Kidney stones: Pathophysiology and medical management. LANCET. 367, 333-344 (2006).

5. Rieu, P. [Infective lithiasis]. Ann Urol (Paris). 39, 16-29 (2005).

6. Ingsathit, A. et al. Prevalence and risk factors of chronic kidney disease in the Thai adult population: Thai SEEK study. Nephrol Dial Transplant. 25, 1567-1575 (2010).

7. Milicevic, S., Bijelic, R., Krivokuca, V., Bojic, M. \& Popovic-Pejicic, S. \& Bojanic, N. Correlation of the body mass index and calcium nephrolithiasis in adult population. Med Arch. 67, 423-427 (2013). 
8. Rendina, D., De Filippo, G., D'Elia, L. \& Strazzullo, P. Metabolic syndrome and nephrolithiasis: A systematic review and meta-analysis of the scientific evidence. J NEPHROL. 27, 371-376 (2014).

9. Liu, L. H. et al. Diabetes mellitus and the risk of urolithiasis: A meta-analysis of observational studies. UROLITHIASIS. 43, 293-301 (2015).

10. Sancak, E. B. et al. Do Hypertension, diabetes mellitus and obesity increase the risk of severity of nephrolithiasis? PAK J MED SCI. 31, 566-571 (2015).

11. Shimizu, T., Hori, H., Umeyama, M. \& Shimizu, K. Characteristics of gout patients according to the laterality of nephrolithiasis: A cross-sectional study using helical computed tomography. INT J RHEUM DIS. 22, 567-573 (2019).

12. Anderson, R. A. A complementary approach to urolithiasis prevention. WORLD J UROL. 20, 294-301 (2002).

13. Sreenevasan, G. Bilateral renal calculi. Ann R Coll Surg Engl. 55, 3-12 (1974).

14. Kelly, C., Geraghty, R. M. \& Somani, B. K. Nephrolithiasis in the obese patient. CURR UROL REP. 20, 36 (2019).

15. Zimmet, P., Magliano, D., Matsuzawa, Y., Alberti, G. \& Shaw, J. The metabolic syndrome: A global public health problem and a new definition. J ATHEROSCLER THROMB. 12, 295-300 (2005).

16. Antonelli, J. A., Maalouf, N. M., Pearle, M. S. \& Lotan, Y. Use of the National Health and Nutrition Examination Survey to calculate the impact of obesity and diabetes on cost and prevalence of urolithiasis in 2030. EUR UROL. 66, 724-729 (2014).

17. Kabeya, Y. et al. Associations of insulin resistance and glycemic control with the risk of kidney stones. Intern Med. 51, 699-705 (2012).

18. Weinberg, A. E., Patel, C. J., Chertow, G. M. \& Leppert, J. T. Diabetic severity and risk of kidney stone disease. EUR UROL. 65, 242-247 (2014).

19. Devasia, D. et al. Association between adiponectin and insulin resistance in diabetic urolithiasis. Oman Med J. 32, 131-134 (2017).

20. Cappuccio, F. P., Strazzullo, P., Farinaro, E. \& Trevisan, M. Uric acid metabolism and tubular sodium handling. Results from a population-based study. JAMA. 270, 354-359 (1993).

21. Taylor, E. N., Stampfer, M. J. \& Curhan, G. C. Diabetes mellitus and the risk of nephrolithiasis. KIDNEY INT. 68, 1230-1235 (2005).

22. Strazzullo, P. et al. Past history of nephrolithiasis and incidence of hypertension in men: A reappraisal based on the results of the Olivetti Prospective Heart Study. Nephrol Dial Transplant. 16, 2232-2235 (2001).

23. Kohjimoto, Y. et al. Association of metabolic syndrome traits and severity of kidney stones: Results from a nationwide survey on urolithiasis in Japan. AM J KIDNEY DIS. 61, 923-929 (2013).

24. Obligado, S. H. \& Goldfarb, D. S. The association of nephrolithiasis with hypertension and obesity: A review. AM J HYPERTENS. 21, 257-264 (2008). 
25. Cho, S. T., Jung, S. I., Myung, S. C. \& Kim, T. H. Correlation of metabolic syndrome with urinary stone composition. INT J UROL. 20, 208-213 (2013).

26. Maalouf, N. M. Metabolic syndrome and the genesis of uric acid stones. J Ren Nutr. 21, 128-131 (2011).

27. Grases, F., Sanchis, P., Perello, J. \& Costa-Bauza, A. Role of uric acid in different types of calcium oxalate renal calculi. INT J UROL. 13, 252-256 (2006).

28. Fellstrom, B. et al. The influence of a high dietary intake of purine-rich animal protein on urinary urate excretion and supersaturation in renal stone disease. Clin Sci (Lond). 64, 399-405 (1983).

29. Wen, J. G. et al. Melamine related bilateral renal calculi in 50 children: Single center experience in clinical diagnosis and treatment. J Urol. 183, 1533-1537 (2010).

30. Zeng, G. et al. Prevalence of kidney stones in China: An ultrasonography based cross-sectional study. BJU INT. 120, 109-116 (2017).

\section{Tables}

Table 1 Demographic characteristics and data of 7587 patients 


\begin{tabular}{|c|c|}
\hline Characteristic & Value \\
\hline $\mathrm{N}$ & 7587 \\
\hline Age(year) & $48.2 \pm 13.1^{*}$ \\
\hline Gender(male) & $6043(79.6)^{\triangle}$ \\
\hline Height(m) & $1.71 \pm 0.07^{*}$ \\
\hline Weight(kg) & $75.6 \pm 12.92^{*}$ \\
\hline $\mathrm{BMI}\left(\mathrm{kg} / \mathrm{m}^{2}\right)$ & $25.96 \pm 3.53^{*}$ \\
\hline \multicolumn{2}{|l|}{ BMI grouping } \\
\hline$\otimes 18.5$ & $101(1.3)^{\triangle}$ \\
\hline$\geq 18.5-\unrhd 24$ & $2072(27.3)^{\triangle}$ \\
\hline$\geq 24-\llbracket 28$ & $3426(45.2)^{\triangle}$ \\
\hline$\geq 28$ & $1988(26.2)^{\triangle}$ \\
\hline Nephrolithiasis (yes) & $2316(30.5)^{\triangle}$ \\
\hline DM(yes) & $1228(16.2)^{\triangle}$ \\
\hline Hypertension(yes) & $2104(27.7)^{\triangle}$ \\
\hline CVD(yes) & $304(4.0)^{\triangle}$ \\
\hline Stroke(yes) & $151(2.0)^{\triangle}$ \\
\hline Liver and gallbladder disease(yes) & $758(10.0)^{\triangle}$ \\
\hline Gastrointestinal disease(yes) & $340(4.5)^{\triangle}$ \\
\hline Respiratory disease(yes) & $591(7.8)^{\triangle}$ \\
\hline Chronic kidney disease(yes) & $197(2.6)^{\triangle}$ \\
\hline Orthopedic disease(yes) & $804(10.6)^{\triangle}$ \\
\hline Tumor disease(yes) & $167(2.2)^{\triangle}$ \\
\hline Gout(yes) & $91(1.2)^{\triangle}$ \\
\hline Other diseases(yes) & $45(0.6)^{\triangle}$ \\
\hline Maximal stone diameter(cm) & $1.05 \pm 0.28^{*}$ \\
\hline
\end{tabular}


Note: (1)*mean \pm standard deviation; $\triangle$ case (\%). (2)BMl=body mass index; $D M=$ diabetes mellitus; $C V D=$ cerebrovascular disease.

Table 2 Characteristics' comparison of the side of the kidney stone (unilateral / bilateral) 


\begin{tabular}{|c|c|c|c|c|}
\hline Characteristics & unilateral & bilateral & $\mathrm{T} / \chi^{2}$ & $P$-Value \\
\hline $\mathrm{N}$ & 4983 & 2604 & & \\
\hline Age(year) & $48.2 \pm 13.2^{*}$ & $48.1 \pm 12.8$ & 0.2902 & $0.7716^{\S}$ \\
\hline Gender(male) & $3945(79.2)^{\triangle}$ & $2089(80.2)$ & 1.1661 & $0.2802^{\ddagger}$ \\
\hline Height(m) & $1.70 \pm 0.07^{*}$ & $1.71 \pm 0.07$ & -1.5185 & $0.1289 \S$ \\
\hline Weight(kg) & $75.3 \pm 12.80^{*}$ & $76.81 \pm 3.10$ & -4.7523 & $<0.0001^{\S}$ \\
\hline $\mathrm{BMI}(\mathrm{kg} / \mathrm{m} 2)$ & $25.82 \pm 3.52^{*}$ & $26.23 \pm 3.53$ & -4.8619 & $<0.0001^{\S}$ \\
\hline BMI grouping $\left(\mathrm{kg} / \mathrm{m}^{2}\right)$ & & & -4.8003 & $<0.0001^{\dagger}$ \\
\hline$\bigotimes 18.5$ & $69(1.4)^{\triangle}$ & $32(1.2)$ & & \\
\hline$\geq 18.5-\llbracket 24$ & $1437(28.8)^{\triangle}$ & $635(24.4)$ & & \\
\hline$\geq 24-\varangle 28$ & $2240(45.0)^{\triangle}$ & $1186(45.5)$ & & \\
\hline$\geq 28$ & $1237(24.8)^{\triangle}$ & $751(28.8)$ & & \\
\hline Nephrolithiasis (yes) & $1334(26.8)^{\triangle}$ & $982(37.7)$ & 96.5207 & $<0.0001^{\ddagger}$ \\
\hline DM(yes) & $758(15.0)^{\triangle}$ & $470(18.0)$ & 11.7137 & $0.0006^{\ddagger}$ \\
\hline Hypertension(yes) & $1309(26.3)^{\triangle}$ & $795(30.5)$ & 15.4913 & $<0.0001^{\ddagger}$ \\
\hline CVD(yes) & $206(4.1)^{\triangle}$ & $98(3.8)$ & 0.6108 & $0.4345^{\ddagger}$ \\
\hline Stroke(yes) & $103(2.1)^{\triangle}$ & $48(1.8)$ & 0.4388 & $0.5077^{\ddagger}$ \\
\hline Liver and gallbladder disease(yes) & $496(10.0)^{\triangle}$ & $262(10.1)$ & 0.0220 & $0.8820^{\ddagger}$ \\
\hline Gastrointestinal disease(yes) & $229(4.6)^{\triangle}$ & $111(4.3)$ & 0.4429 & $0.5057^{\ddagger}$ \\
\hline Respiratory disease(yes) & $400(8.0)^{\triangle}$ & 191(7.3) & 1.1416 & $0.2853^{\ddagger}$ \\
\hline Chronic kidney disease(yes) & $128(2.6)^{\triangle}$ & $69(2.6)$ & 0.0444 & $0.8331^{\ddagger}$ \\
\hline Orthopedic disease(yes) & $532(10.7)^{\triangle}$ & $272(10.4)$ & 0.0961 & $0.7564^{\ddagger}$ \\
\hline Tumor disease(yes) & $110(2.2)^{\triangle}$ & $57(2.2)$ & 0.0027 & $0.9582^{\ddagger}$ \\
\hline Gout(yes) & $38(0.8)^{\triangle}$ & $53(2.0)$ & 23.3781 & $<0.0001^{\ddagger}$ \\
\hline Other diseases(yes) & $32(0.6)^{\triangle}$ & $13(0.5)$ & 0.5918 & $0.4413^{\ddagger}$ \\
\hline
\end{tabular}




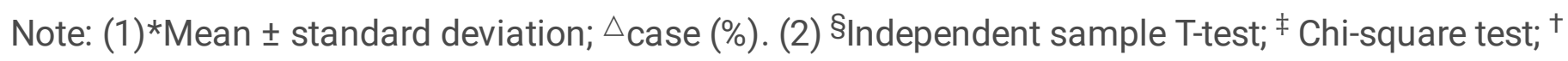
Mann-Whitney U test. (3) BMI=Body mass index; DM= Diabetes mellitus; $C V D=$ Cerebrovascular disease.

Table 3 Characteristics' comparison of the side of stone (left / right / bilateral) 


\begin{tabular}{|c|c|c|c|c|c|}
\hline Characteristics & left & right & bilateral & $\mathrm{F} / \chi^{2}$ & $P$-Value \\
\hline $\mathrm{N}$ & 2719 & 2264 & 2604 & & \\
\hline Age(year) & $47.9 \pm 13.1^{*}$ & $48.5 \pm 13.3$ & $48.1 \pm 12.8$ & 1.4765 & $0.2285^{\S}$ \\
\hline Gender(male) & $2157(79.3)^{\triangle}$ & 1788(79.0) & $2089(80.2)$ & 1.2620 & $0.5321^{\ddagger}$ \\
\hline Height(m) & $1.71 \pm 0.07^{*}$ & $1.70 \pm 0.07$ & $1.71 \pm 0.07$ & 1.7616 & $0.1718^{\S}$ \\
\hline Weight(kg) & $75.1 \pm 12.8^{\star}$ & $75.5 \pm 12.8$ & $76.81 \pm 3.10$ & 11.8905 & $<0.0001^{\S}$ \\
\hline $\mathrm{BMI}\left(\mathrm{kg} / \mathrm{m}^{2}\right)$ & $25.72 \pm 3.49^{\star}$ & $25.94 \pm 3.56$ & $26.23 \pm 3.53$ & 14.2400 & $<0.0001^{\S}$ \\
\hline BMI grouping $\left(\mathrm{kg} / \mathrm{m}^{2}\right)$ & & & & 29.1423 & $<0.0001^{\dagger}$ \\
\hline$\bigotimes 18.5$ & $40(1.5)^{\triangle}$ & $29(1.3)$ & $32(1.2)$ & & \\
\hline$\geq 18.5-\llbracket 24$ & $803(29.5)^{\triangle}$ & $634(28.0)$ & $635(24.4)$ & & \\
\hline$\geq 24-\llbracket 28$ & $1244(45.8)^{\triangle}$ & $996(44.0)$ & $1186(45.6)$ & & \\
\hline$\geq 28$ & $632(23.2)^{\triangle}$ & $605(26.7)$ & $751(28.8)$ & & \\
\hline Nephrolithiasis (yes) & $751(27.6)^{\triangle}$ & $583(25.8)$ & $982(37.7)$ & 98.5567 & $<0.0001^{\ddagger}$ \\
\hline DM(yes) & $409(15.0)^{\triangle}$ & $339(15.0)$ & $470(18.0)$ & 11.7180 & $<0.0001^{\ddagger}$ \\
\hline Hypertension(yes) & $698(25.7)^{\triangle}$ & $611(27.0)$ & $795(30.5)$ & 16.5595 & $<0.0001^{\ddagger}$ \\
\hline CVD(yes) & $111(4.1)^{\triangle}$ & $95(4.2)$ & $98(3.8)$ & 0.6523 & $0.8216^{\ddagger}$ \\
\hline Stroke(yes) & $56(2.1)^{\triangle}$ & $47(2.1)$ & $48(1.8)$ & 0.4405 & $0.8023^{\ddagger}$ \\
\hline $\begin{array}{l}\text { Liver and gallbladder } \\
\text { disease(yes) }\end{array}$ & $278(10.2)^{\triangle}$ & $218(9.6)$ & $262(10.1)$ & 0.5090 & $0.7753^{\ddagger}$ \\
\hline Gastrointestinal disease(yes) & $111(4.1)^{\triangle}$ & $118(5.2)$ & $111(4.3)$ & 4.1256 & $0.1271^{\ddagger}$ \\
\hline Respiratory disease(yes) & $209(7.7)^{\triangle}$ & 191(8.4) & 191(7.3) & 2.1083 & $0.3485^{\ddagger}$ \\
\hline Chronic kidney disease(yes) & $73(2.7)^{\triangle}$ & $55(2.4)$ & $69(2.6)$ & 0.3632 & $0.8339^{\ddagger}$ \\
\hline Orthopedic disease(yes) & $285(10.5)^{\triangle}$ & $247(10.9)$ & $272(10.4)$ & 0.3351 & $0.8457^{\ddagger}$ \\
\hline Tumor disease(yes) & $60(2.2)^{\triangle}$ & $50(2.2)$ & $57(2.2)$ & 0.0028 & $0.9986^{\ddagger}$ \\
\hline Gout(yes) & $21(0.8)^{\triangle}$ & $17(0.8)$ & $53(2.0)$ & 23.3828 & $<0.0001^{\ddagger}$ \\
\hline Other diseases(yes) & $20(0.7)^{\triangle}$ & $12(0.5)$ & $13(0.5)$ & 1.4778 & $0.4776^{\ddagger}$ \\
\hline
\end{tabular}




\begin{tabular}{|c|c|c|c|c|c|}
\hline Maximal stone diameter(cm) & $1.03 \pm 0.27^{*}$ & $1.02 \pm 0.27$ & $1.09 \pm 0.29$ & 47.4977 & $<0.0001^{\S}$ \\
\hline Number of stones $₫$ case $\rrbracket$ & & & & 2.6460 & $0.1040^{\ddagger}$ \\
\hline Single & $1649(60.6)^{\triangle}$ & $1424(62.9)$ & & & \\
\hline Multiple & $1070(39.4)^{\triangle}$ & $840(37.1)$ & & & \\
\hline
\end{tabular}

Note: (1)^Mean \pm standard deviation; $\triangle$ case (\%). (2) §one-way ANOVA; ${ }^{\ddagger}$ Chi-square test; ${ }^{\dagger}$ Kruskal-Wallis $\mathrm{H}$ Test. (3) $\mathrm{BMI}=$ Body mass index; $\mathrm{DM}=$ Diabetes mellitus; $\mathrm{CVD}=$ Cerebrovascular disease.

Table 4 The logistic regression results of univariate and multivariate analysis

\begin{tabular}{|lllllll|}
\hline Characteristics & \multicolumn{5}{c|}{ Multivariate analysis } \\
& \multicolumn{1}{c|}{ OR } & $95 \% \mathrm{Cl}$ & P-Value & OR & $95 \% \mathrm{Cl}$ & P-Value \\
\hline BMI $\left(\mathrm{kg} / \mathrm{m}^{2}\right)$ & 1.03 & $(1.02,1.05)$ & $<0.0001$ & 1.03 & $(1.02,1.04)$ & $<0.0001$ \\
\hline Nephrolithiasis & 1.66 & $(1.50,1.83)$ & $<0.0001$ & 1.56 & $(1.41,1.73)$ & $<0.0001$ \\
\hline DM & 1.25 & $(1.10,1.42)$ & 0.0006 & 1.14 & $(1.00,1.30)$ & 0.0457 \\
\hline Hypertension & 1.23 & $(1.11,1.37)$ & $<0.0001$ & 1.15 & $(1.03,1.28)$ & 0.0129 \\
\hline Gout & 2.70 & $(1.78,4.11)$ & $<0.0001$ & 2.50 & $(16.4,3.83)$ & $<0.0001$ \\
\hline Maximal stone diameter(cm) & 2.29 & $(1.93,2.73)$ & $<0.0001$ & 2.07 & $(1.74,2.47)$ & $<0.0001$ \\
\hline
\end{tabular}

Note: (1)BMI=body mass index; $\mathrm{DM}=$ diabetes mellitus. (2)OR=odds ratio; $\mathrm{Cl}=$ confidence interval. 